\title{
28 Research Suare \\ Practices of Sickle Cell Disease Genetic Screening and Testing in the Prenatal Population
}

Arianna Prince ( $\square$ aprince14@gwu.edu )

The George Washington School of Medicine and Health Sciences

\section{Alanna Cruz-Bendezú}

The George Washington School of Medicine and Health Sciences

Nicole Gunawansa

The George Washington School of Medicine and Health Sciences

Jenna Wade

The George Washington School of Medicine and Health Sciences

Victoria H. Coleman-Cowger

Emmes Corporation

Jay Schulkin

Pregnancy-Related Care Research Network

Charles Macri

Department of Obstetrics and Gynecology, George Washington University Medical Faculty Associates

\section{Research Article}

Keywords: Genetic Screening, Sickle Cell, Provider practices

Posted Date: October 7th, 2021

DOI: https://doi.org/10.21203/rs.3.rs-929284/v1

License: (c) (i) This work is licensed under a Creative Commons Attribution 4.0 International License.

Read Full License 


\section{Abstract \\ Background}

Genetic screening and testing has only recently become an accessible assessment for genetic risk. Unfortunately, these technologies have been underutilized in minority populations despite their usefulness for predicting diseases like sickle cell disease (SCD), often found in African American and Black and Hispanic populations. We surveyed prenatal patients to understand current choices, beliefs and experiences surrounding genetic screening and testing, specifically for sickle cell disease.

\section{Methods}

In this cross-sectional survey, we collected information from 322 women during prenatal visits from July 2019 through May 2021. Responses to questions about pregnancy screening and testing practices were analyzed for trends to identify barriers to care and education about testing and screening for sickle cell disease. Patients were asked to rate whether they agree or disagree with statements regarding sickle cell health behaviors. We used $\chi^{2}$ tests to compare categorical variables by self-reported race. Binary logistic regression was used to determine the odds ratios and confidence intervals for each outcome.

\section{Results}

Women were a mean (SD) age of 33.3 (6.1). $42.9 \%$ of patients self-identified as White while 41.3 of patients self- identified as African American/Black. Screening questions were adjusted for differences in race, insurance, and education levels to show significant differences in responses between African Americans/Blacks and Whites for screening for SCD ( $p=0.047,0 R 95 \% \mathrm{Cl}=0.455$ [0.210-0.989]) and plans to meet with genetic counselors $(p=0.049, \mathrm{OR} 95 \% \mathrm{Cl}=0.299$ [0.090-0.993]). Regarding sickle cell health behaviors, only the responses to the statements about if sickle cell is not in their family, then it is likely not in themselves or their children, and partners' opinions about genetic screening remained significant $(p=0.011$ and $p=0.081$, respectively).

\section{Conclusions}

Our findings suggest gaps in screening, testing, and educational efforts between African American/Black and White patients, as well as differences in opinions regarding pregnancy management with a family history or gestational diagnosis of sickle cell disease. Future research should focus on decreasing these healthcare gaps and improving education that address concerns about SCD for relevant populations.

\section{Background:}


Sickle cell disease is a group of inherited blood disorders that primarily affects populations of Black or African American descent. The most severe form of SCD is HbSS, or sickle cell anemia. People who have this type of SCD inherit two sickle cell ("S") genes, one from each parent, whereas people who have sickle cell trait (SCT) inherit one " $S$ " gene from one parent and one healthy gene (" $A$ ") from the other parent. The potential for SCT or SCD can be examined in two manners. Genetic screening usually requires a blood sample, and presents the likelihood of a chromosomal condition in the pregnancy, but it is not diagnostic. Genetic testing is performed either as chorionic villus sampling (CVS) or amniocentesis and is diagnostic for chromosomal disorders. Placental tissue or amniotic fluid is taken, respectively, and the fetus' chromosomes are analyzed.

According to the CDC (2020), SCD occurs in 1 in every 365 Black and African American births, and 1 per 16,300 Hispanic-American births (1). SCT occurs in 1 in every 13 Black or African American births. In addition to long-term health complications, including acute chest syndrome, stroke, infection, and organ failure, SCD is estimated to have an annual cost per patient ranging from $\$ 18,859$, between ages $0-9$, to peak costs of $\$ 43,586$, for ages 20-29 (2). The decline in annual costs with increasing age may be related to increasing complications leading to morbidity and mortality.

SCD is also a historically underfunded orphan disease, in comparison to other orphan diseases, such as Cystic Fibrosis, which affects half the number of people, comparatively, in the United States (3). SCD has fewer comprehensive health care centers and specialized providers. Despite SCD newborn screens being implemented in 2006, SCD patients have decreased counseling and prophylactic treatments compared to other chronic diseases, increasing the risk of cognitive complications and death in sickle cell patients. Inequalities in treatment are also linked to $60 \%$ of SCD patients using Medicare and Medicaid for insurance, limiting access to specialized care, decreased use of primary care providers and increased emergency department visits, and stigmas surrounding pain relief in patients with sickle cell pain crises. Though there are still limited SCD treatments and research remains underfunded in comparison to Cystic Fibrosis, there have been recent efforts to identify cures for sickle cell disease via genetic therapies, and The Cure Sickle Cell Initiative was formed by the National Heart, Lung, and Blood Institute in 2017 to accelerate the identification of these genetic-based cures for SCD (4).

Within the Ashkenazi American and Canadian Jewish population, the incidence of Tay Sachs Disease (TSD) has been reduced by $90 \%$ (5). This reduction in TSD cases has, in large part, been due to extensive prenatal screening, education and counseling. While this attempt to eliminate TSD provides numerous ethical, privacy, and communication considerations, few diseases have been voluntarily eliminated as successfully as TSD. Until now, modern genetic screening and testing, as well as education, has not been as extensively researched and offered to minority populations, including Black and African American patients at risk for SCD (6).

Additionally, the perceived burden of SCD may affect prenatal genetic testing and screening choices. In a study performed in Cameroon, the majority of parents with children suffering from SCD would choose to abort the fetus if it was found to be affected. However, these may not reflect the views of parents in 
industrialized countries, where access to healthcare has a relatively reasonable prognosis and quality of life. In a United States study that conducted focus groups for adults living with SCD or SCT, it was found that reproductive decisions were largely based on previous experiences of suffering and the severity associated with the disease (7). However, these decisions were complicated by desires to have children and faith in modern treatments that increase longevity. Additionally, for some, attitudes towards genetic testing, screening and abortion, were influenced by religious and/or personal moral judgement, values and preferences. The general beliefs of the adults in the study was that knowing one's trait status is important and trait status should be discussed with partners to prevent transmission. However, the participants acknowledged the difficulty of sharing one's SCT status with a partner, and that testing resistance was further compounded by partners' fear of the pain associated with phlebotomy, and convincing partners of the need to test. In similar studies, African American women have been shown to believe that SCD is severe enough to warrant screening, there is benefit for SCT testing, and that barriers to screening are low. However, there was low perception of personal susceptibility to having children that carry the SCT or SCD.

Disparities in genetic testing are further compounded since African Americans, Latinos and non-Hispanic Whites have been shown to have differences in knowledge about genetic testing and types of health insurance, and significant concerns about the misuse of genetic material and mistrust in physicians and the medical system (8). In regards to SCD and SCT, this is not unfounded. The National Sickle Cell Anemia Control Act of 1972 was an attempt to increase healthcare for those with SCT. Instead, it resulted in job and insurance discrimination for these patients (9). This example further highlights the need for proper and ethical communication and education regarding SCD and potential benefits of genetic testing and screening.

Choices regarding prenatal diagnosis of SCD and termination are also complicated. According to the American Society of Hematology, the disease manifests variably and there is no prenatal prediction of severity, with patients in industrialized countries living, on average, to greater than 40 years (10). Additionally, there are variable beliefs among Black and African American communities about abortion related to education, income, religiousness, and location demographics (11). However, it was found that overall Blacks and African Americans have a lower degree of support for abortion than Whites. In the most recent data about "The State of Abortion and Contraception Attitudes in All 50 States," the majority of African Americans generally support abortion as a contraceptive method while Hispanic Americans are slightly more opposed to abortion (12).

The goal of this research is to investigate current educational, as well as genetic testing and screening practices surrounding SCD in prenatal populations. Alongside previously identified barriers discussed in the literature, this research will help identify barriers to knowledge and testing, and determine potential areas for healthcare providers to counsel and educate patients about SCD, reproductive decisions, and care, to decrease inequalities in care for SCD patients.

\section{Methods:}




\section{Patients and Procedures}

Participants included 500 women attending either prenatal or gynecological appointments at the George Washington Medical Faculty Associates (GW MFA) offices in Washington, DC. Data was collected between July 2019 and May 2021. Participants were at least 18 years of age and were competent enough in English to read information sheets about the survey and respond to the survey questions. The study was approved by the George Washington University Committee on Human Research, Institutional Review Board.

Patients were recruited by George Washington School of Medicine and Health Sciences students within the GW MFA obstetrics and gynecology (Ob/Gyn) offices. Women were randomly approached either in the waiting room or in between having their vitals taken and speaking with the nurse, and the doctor entering the room. Patients were explained the nature of the study, asked if they believed they qualified as primarily a prenatal or gynecological patient, and were given privacy to fill out either the prenatal or gynecological survey, based on their previous answer. Any patient questions about the survey were answered by the recruiter. If patients incorrectly chose the gynecological or prenatal study, their data was not included in the 500 participants. All surveys were placed in confidential envelopes and stored in a locked cabinet only accessed by study researchers. Data was stored on a password protected Excel document on password protected computers. All survey questions were voluntary and patient information was non-identifiable to a specific patient.

\section{Prenatal Survey:}

\section{Sociodemographic Information}

These questions asked about year the patient was born, health insurance coverage, racial and ethnic group (American Indian or Alaska Native, Black or African American, Asian, Native Hawaiian/Other Pacific Islander, Hispanic or Latino, and White) and highest degree achieved (Grade School, High School/GRD, College Degree or Graduate Degree)

\section{Pregnancy and Appointment Information}

Patients were asked the reason for their appointment at the MFA Ob/Gyn Clinic and how many weeks along they were in their pregnancy. They were also asked if they were previously pregnant, and if so, had they done genetic screening or diagnostic testing, and whether they had discussed genetic screening or diagnostic testing with their provider in the current pregnancy.

\section{Family History}

The survey asked patients about their knowledge of their family history of sickle cell anemia, if they ever were told they had anemia, and if so, what type of anemia. 
Patients were asked if they had been screened for sickle cell anemia, if the provider explained the difference between screening and testing for sickle cell anemia. Patients were then asked to rate the degree the healthcare provider answered their questions about screening and tests. Response options included "unable to answer any of my questions," "answered some, but not all my questions," and "answered all my questions." Similarly, patients were asked about plans to meet with a genetic counselor to discuss sickle cell anemia or if they had received counseling for sickle cell anemia. Similarly, patients were asked to rate the degree they felt their questions were answered using the same response options.

\section{Attitudes Towards Sickle Cell and Pregnancy Management}

Patients were asked to evaluate their attitudes about statements regarding family history, partner opinions, and managing a pregnancy with a sickle cell anemia positive genetic test. (For example "If sickle cell is not in my family, then I do not think it likely in me or my children.") Responses were assessed on a Likert scale ranging from Strongly Agree $=1$ to Strongly Disagree $=5$.

\section{Information sheet and Usefulness}

Patients were given a prenatal information sheet about sickle cell anemia and asked to evaluate its usefulness. (Response options included “Not Useful," “Useful," or “Very Useful”).

\section{Data Analysis:}

All data from surveys was entered into a Google sheet. Data was analyzed using SPSS software for statistical computing and descriptive statistics. We used $\chi^{2}$ tests to compare categorical variables by race self-reporting. Binary logistic regression was used to determine the odds ratios and $95 \%$ confidence intervals for each of the outcome variables. $P$ value significance was set at $\leq 0.05$. The data was adjusted solely for race (Model 1), and race, insurance, and education (Model 2). P values and odds ratios were generated for each of the adjusted data categories. In regards to data analysis of the Likert scale, data was adjusted to either Agree (Strongly Agree and Agree) or Not Agree (Neutral, Disagree, Strongly Disagree). Similarly, education level grouped college graduates and graduate/professional degree as one category of "college degree or more."

\section{Results:}

A total of $322 / 500$ patient surveys were analyzed (Table 1 ). 
Table 1

Prenatal Maternal Characteristics and Exposures by Race

\begin{tabular}{|c|c|c|c|}
\hline & Overall & Black/AA & White \\
\hline & $N=322$ & $N=132$ & $N=137$ \\
\hline \multicolumn{4}{|l|}{ Participant Characteristics N (\%) } \\
\hline Average Age M (SD) & $33.3(6.3)$ & & \\
\hline \multicolumn{4}{|l|}{ Race/Ethnicity (\%) } \\
\hline White & $137(42.9)$ & & \\
\hline Black or African American & $132(41.3)$ & & \\
\hline American Indian or Alaska Native & $7(2.2)$ & & \\
\hline Asian & $18(5.6)$ & & \\
\hline Native Hawaiian or other Pacific Islander & $1(0.3)$ & & \\
\hline Hispanic or Latino & $15(4.7)$ & & \\
\hline Other & $12(3.7)$ & & \\
\hline \multicolumn{4}{|l|}{ Education } \\
\hline Less than high school diploma & $5(1.5)$ & 2 & 0 \\
\hline High school diploma, GED, or equivalent & $99(30.7)$ & 89 & 3 \\
\hline College Graduate & $71(22)$ & 20 & 38 \\
\hline Graduate/Professional Degree & $146(45.3)$ & 20 & 96 \\
\hline \multicolumn{4}{|l|}{ Number of pregnancy } \\
\hline First one & $120(37.3)$ & 35 & 60 \\
\hline Previous Pregnancy & $202(62.7)$ & 97 & 77 \\
\hline \multicolumn{4}{|l|}{ Health Insurance Status } \\
\hline Yes & $310(96.2)$ & $127(96.2)$ & 134 (97.8) \\
\hline No & $12(3.7)$ & $5(3.8)$ & $3(2.8)$ \\
\hline
\end{tabular}

Surveys were not analyzed if most of the survey was incomplete or patients completed a gynecological survey. Patient surveys were also excluded from analysis if the patient did not identify as White or Black/African American since there was not a large enough sample size from Asian, American Indian or Alaska Native, Native Hawaiian/ Other Pacific Islander or the Hispanic/Latino populations to generate significant data. 137 (42.9\%) of patients self-identified as White, and 132 patients (41.3\%) of patients self- identified as African American/Black. The age range for patients was between 20 years and 57 
years, with the average patient age of 33.3 years. Patients ranged from 4 to 41 weeks pregnant with the average patient 24.6 weeks pregnant. For $37.3 \%$ of patients, this was their first pregnancy and $96.2 \%$ have health insurance. Finally, $1.5 \%$ of patients have less than a high school diploma, $30.7 \%$ of patients have a high school diploma, GED, or equivalent, $22 \%$ of patients are college graduates, and $45.3 \%$ of patients have a graduate or professional degree.

Most patients reported that they had not been screened for SCD in their current pregnancy $(73 \%)$ (Table 2).

Table 2

Prenatal Responses to Screening/Testing Experience

\begin{tabular}{|lccc|}
\hline \multicolumn{4}{|l|}{ Measured Outcomes N (\%) } \\
\hline Screened for Sickle Cell Anemia & & & \\
\hline Yes & $235(27.0)$ & 44 & 24 \\
\hline No & $233.0)$ & 88 & 113 \\
\hline Explained difference between testing/screening & & \\
\hline Yes & $58(18.0)$ & 31 & 14 \\
\hline No & $255(79.2)$ & 98 & 120 \\
\hline Plans to meet with genetic counselor & & & \\
\hline Yes & $33(10.2)$ & 24 & 6 \\
\hline No & $267(82.9)$ & 101 & 122 \\
\hline
\end{tabular}

There was a significant difference $(p=0.003)$ between African American/Black and White populations, with African American/Black patients being less likely to report being screened for SCD than White patients (OR 95\% Cl=0.425 [0.240-0.751]). The majority did not think their provider had explained the difference between genetic testing and screening (79.2\%). There was a significant difference $(p=0.005)$ between African American/Black and White populations, with African American/Black patients being less likely to report having the difference in genetic testing/screening explained than White patients (OR 95\% $\mathrm{Cl}=0.373$ [0.188-0.740]) Only $10.2 \%$ of patients had plans to meet with a genetic counselor. Of this population, African Americans/Blacks were significantly $(p=0.001)$ less likely to plan to meet with a genetic counselor than White patients (OR 95\% $\mathrm{Cl}=0.207$ [0.081-0.526]).

When the responses to the three questions about screening for SCD, differences in genetic testing and screening, and plans to meet with a genetic counselor were adjusted for differences in race, insurance and education levels, there were still significant differences in responses between African Americans/Blacks and Whites for screening for SCD ( $p=0.047,0 R 95 \% \mathrm{Cl}=0.455$ [0.210-0.989]) and plans to meet with genetic counselors $(p=0.049,0 R 95 \% \mathrm{Cl}=0.299$ [0.090-0.993]). 
Patients were asked to rate whether they agree or disagree with a statement that if sickle cell is not in their family, then it is likely not in themselves or their children. The majority of patients (50.6\%) agreed with this statement, with $40.1 \%$ not agreeing, and $9.3 \%$ choosing not to answer (Table 3).

Table 3

Level of Agreement with Statements about Sickle Cell, Pregnancy and Partner Wishes

\begin{tabular}{|llll|}
\hline Statements $\mathbf{N}$ (\%) & & $\mathrm{N}=132$ & $\mathrm{~N}=137$ \\
\hline Sickle Cell in family & $163(50.6)$ & $42(31.8)$ & $90(65.7)$ \\
\hline Agree & $129(40.1)$ & $82(62.1)$ & $31(22.6)$ \\
\hline Not Agree & & & \\
\hline Managing Pregnancy & $44(13.7)$ & $17(12.9)$ & $23(16.8)$ \\
\hline Agree & $247(76.7)$ & $104(78.8)$ & $99(72.3)$ \\
\hline Not Agree & & & \\
\hline Termination of Pregnancy & $206(64.0)$ & $97(7.3)$ & $79(57.7)$ \\
\hline Agree & $78(24.2)$ & $23(17.4)$ & $40(29.2)$ \\
\hline Not Agree & & & \\
\hline Partner and Sickle Cell & $15(4.7)$ & $11(8.3)$ & $2(1.5)$ \\
\hline Agree & $271(84.2)$ & $110(83.3)$ & $118(86.1)$ \\
\hline Not Agree & & & \\
\hline Late in Pregnancy & $38(11.8)$ & $12(9.1)$ & $19(13.9)$ \\
\hline Agree & $239(74.2)$ & $107(81.1)$ & $97(70.8)$ \\
\hline Not Agree & &
\end{tabular}

There was a significant difference in response to this statement between African American/Blacks and White populations, with African Americans/Blacks more likely to agree with this statement $(p<0.0001,0 R$ $95 \% \mathrm{Cl}=5.668$ [3.263-9.846]). The majority of patients (76.7\%) disagreed with the statement that they would not use information from SCD screens/tests to manage their pregnancies, with no significant differences in African American/Black and White populations.

About $64 \%$ of patients agreed with the statement that they would not terminate their pregnancy for a SCD diagnosis. There was a significant $(p=0.009)$ difference in African American/Black and White response to this question, with African Americans/Blacks more likely to disagree with this statement and consider termination (OR 95\% $\mathrm{Cl}=0.448$ [0.246-0.815]). Most patients disagreed with the statement that their partner did not want them to get sickle cell screening, also with significant $(p=0.03)$ differences between 
African American/Black and White populations (OR 95\% Cl= 0.181 [0.039-0.846]). African American/Blacks were more likely to have partners that wanted them to be screened. Finally, the majority $(74.2 \%)$ of patients disagreed with the statement that they were too far along in their pregnancy to have genetic testing, with no significant differences between African American/Black and White populations. After adjusting for race and education levels, only responses to the statements about if sickle cell is not in their family, then it is likely not in themselves or their children, and partners' opinions about genetic screening remained significant $(p=0.011$ and $p=0.081$, respectively).

\section{Discussion:}

Overall, we found that the most significant differences between races are between approaches to education, testing/screening, and beliefs and practices surrounding a gestational diagnosis of SCD. Despite SCD predominantly affecting the African American/Black population, African Americans/Blacks reported that they were less likely to be screened than Whites for SCD and were less likely to meet with genetic counselors. Furthermore, African Americans/Black patients reported less frequency of providers explaining the differences between genetic screening and testing. This information is particularly critical since the vast majority of people with SCD are African American/Black patients. Considering the severity of SCD, these patients should be screened, educated, and meet with genetic counselors at least equally, if not increasingly, compared to their White counterparts. It is important to note, that these significant differences remained, even when adjusted by education and insurance, showing that it is not a difference in insurance coverage or education level that is causing differences in screening, knowledge base, and usage of genetic counselors. However, the quality and coverage of the insurance is not listed, which may affect these answers and the data was heavily skewed to a patient population with both relatively high insurance coverage and education level.

Similarly, we saw differences across races regarding patient knowledge of family history, and usage of genetic testing/screening to manage their pregnancies. Regarding the African American/Black patients being more likely to agree with the statement "If sickle cell is not in my family, then I do not think it likely in me or my children," this difference may be contributed to personal family history with SCD or reflect patient knowledge about a disease particularly relevant to their own race/ethnicity. Personal experience of the pain and suffering associated with the SCD might also influence African American/Black patients' greater likelihood of considering terminating a fetus with a SCD diagnosis, over White patients. Interestingly, in contrast to previous studies, we found that African American/Black patients' partners overall supported SCD screening, in contrast to previous studies which contradicted this (13). This increase in support may be associated with increased educational efforts by Ob/Gyn practices about the importance of genetic screening.

Limitations to this study include that patient surveys were voluntary, based on convenience sampling, and based on patient self-identification of race. As there is no standardized patient sample to compare the study population and clinic population, this study may not be generalizable to all patients, especially patients in other populations that have different demographics. However, a strength of the study is that 
patients were randomly sampled between 8 am- 5 pm regardless of reason for their prenatal visit which increases the likelihood of generalizability. Additionally, patients were required to answer on a binary agree or disagree without room for explanations and data is self-reported so any recall bias may skew results.

\section{Conclusions:}

The study findings suggest that African American/Black patients experience disparities in education and genetic screening and testing practices compared with White patients. Additionally, African American/Black patients have distinctive opinions about family history and pregnancy management based on a SCD diagnosis. This suggests that providers need to increase their educational and testing/screening efforts in the African American/Black populations, particularly about SCD. In addition, SCD educational practices should be updated to reflect concerns or opinions for relevant populations, in this case, African American/Black populations. Future research should focus on studies assessing the effectiveness of educational tools that address concerns about SCD specific to the African American/Black populations as well as a standardized protocol for screening and testing patients at risk for SCD.

\section{Abbreviations:}

$\mathrm{Cl}=$ Confidence Interval

CVS $=$ Chorionic Villus Sampling

GW MFA = GW Medical Faculty Associates

Ob/Gyn = Obstetrics and Gynecology

$\mathrm{OR}=$ Odds ratio

SCD = Sickle Cell Disease

SCT $=$ Sickle Cell Trait

SD = Standard Deviation

TSD = Tay Sachs Disease

\section{Declarations:}

Ethics Approval and consent to participate. All methods were performed in accordance with the relevant guidelines and regulations set forth in the Declaration of Helsinki as well as the George Washington University Office of Human Research. A fact sheet and information about the study was provided to 
women at their prenatal visit and informed consent was obtained from the participants, before they completed the survey. The study protocol, including ethic committee approval for the study, was approved by The George Washington University Committee on Human Research Institutional Review Board, FWA00005945.

Consent for publication: Not applicable.

Availability of data and materials: The datasets used during the current study are available from the corresponding author on reasonable request.

Competing Interests: The authors declare that they have no competing interests.

Funding: No funding to be declared for this study.

Authors' contributions: AP, ACB, NG, and JW conceptualized and designed the study, analyzed, and interpreted the data, and drafted the initial manuscript. CM, VCC, JS, and JW interpreted the data and critically reviewed the manuscript for important intellectual content.

All authors read and approved the final manuscript.

Acknowledgements. JS is supported by a grant from the Health Resources and Services Administration UA6MC31609 which supports the Pregnancy-Related Care Research Network.

\section{References:}

1. Sickle Cell Disease (SCD): Center for Disease Control and Prevention; [updated December 16, 2020. Available from: https://www.cdc.gov/ncbddd/sicklecell/data.html.

2. Salcedo J, Young CM, Bulovic J. The Total Direct Cost of Healthcare in the United States in Patients with Sickle Cell Disease: A Propensity Score Matched Analysis. Blood. 2019;134:4671.

3. Lee L, Smith-Whitley K, Banks S, Puckrein G. Reducing Health Care Disparities in Sickle Cell Disease: A Review. Public Health Rep. 2019;134(6):599-607.

4. Bahr NC, Song J. The Effect of Structural Violence on Patients with Sickle Cell Disease. J Health Care Poor Underserved. 2015;26(3):648-61.

5. Kaback MM. Population-based genetic screening for reproductive counseling: the Tay-Sachs disease model. Eur J Pediatr. 2000;159 Suppl 3:S192-5.

6. Reed-Weston AE, Espinal A, Hasar B, Chiuzan C, Lazarin G, Weng C, et al. Choices, attitudes, and experiences of genetic screening in Latino/a and Ashkenazi Jewish individuals. J Community Genet. 2020;11(4):391-403.

7. Gallo AM, Wilkie D, Suarez M, Labotka R, Molokie R, Thompson A, et al. Reproductive decisions in people with sickle cell disease or sickle cell trait. West J Nurs Res. 2010;32(8):1073-90. 
8. Suther S, Kiros GE. Barriers to the use of genetic testing: a study of racial and ethnic disparities. Genet Med. 2009;11(9):655-62.

9. Thompson HS, Valdimarsdottir HB, Jandorf L, Redd W. Perceived disadvantages and concerns about abuses of genetic testing for cancer risk: differences across African American, Latina and Caucasian women. Patient Educ Couns. 2003;51(3):217-27.

10. Rare Patients With Sickle Cell Disease Live Nearly Twice as Long as Average [press release]. 2016.

11. Combs MW, Welch S. Blacks, whites, and attitudes towards abortion. Public Opin Q. 1982;46(4):51020.

12. Jones RP, Jackson N, Najle M, Bola O, Greenberg D. The State of Abortion and Contraception Attitudes in All 50 States: Public Religion Research Institute; 2019 [Available from: https://www.prri.org/research/legal-in-most-cases-the-impact-of-the-abortion-debate-in-2019america/.

13. Gustafson SL, Gettig EA, Watt-Morse M, Krishnamurti L. Health beliefs among African American women regarding genetic testing and counseling for sickle cell disease. Genet Med. 2007;9(5):30310. 